\title{
Paclitaxel Coating Inhibits Inflammation Surrounding Subcutaneously Implanted Expanded Polytetrafluoroethylene (ePTFE) Hemodialysis Grafts in Rabbit Model
}

\author{
Insu Baek, Yu-Ji Lee, ${ }^{\dagger}$ Soo Jin Park, ${ }^{\dagger}$ Cheng Zhe Bai, Jong-sang Park, and Dae Joong Kim ${ }^{\dagger}{ }^{*}$ \\ School of Chemistry \& Molecular Engineering, Seoul National University, Seoul 151-742, Korea \\ ${ }^{\dagger}$ Division of Nephrology, Samsung Medical Center, Sungkyunkwan University School of Medicine, Seoul 135-710, Korea \\ *E-mail: kimdjsmc@dreamwiz.com \\ Received December 8, 2009, Accepted December 10, 2009
}

\begin{abstract}
Hemodialysis vascular access dysfunction (HVAD) due to the aggressive development of venous neointimal hyperplasia remains a major complication for patients with synthetic arteriovenous grafts. Paclitaxel-coated expanded polytetrafluoroethylene (ePTFE) grafts effectively prevent neointimal hyperplasia and stenosis. However, perigraft inflammation or edema can be another complication of ePTFE grafts, preventing early cannulation. Three different types of ePTFE grafts, including grafts without paclitaxel coating (control group, $\mathrm{n}=12$ ), grafts with paclitaxel coating at a dose density of $0.61 \mathrm{ug} / \mathrm{mm}^{2}$ (low concentration group, $\mathrm{n}=12$ ), and grafts with paclitaxel coating at a dose density of $1.15 \mathrm{ug} / \mathrm{mm}^{2}$ (high concentration group, $\mathrm{n}=12$ ) were placed in the backs of 12 rabbits, simultaneously. Six rabbits were euthanized after one week and the remaining six were euthanized two weeks after implantation. Perigraft inflammation, graft wall inflammation, stromal cell proliferation, blood vessel formation, tissue necrosis and edema were analyzed for the grafts in each animal. Inflammation surrounding the paclitaxel-coated grafts was significantly reduced compared to the control group. Stromal cell layers were detected at the interface between the graft and the surrounding tissue in the control group, infiltrated into the graft interstices, and differentiated into myofibroblasts for graft healing. Paclitaxel-coated grafts inhibited stromal cell proliferation and infiltration into the graft wall. Tissue necrosis and edema were not detected in either of the paclitaxel-coated graft groups.
\end{abstract}

Key Words: ePTFE, Graft, Inflammation, Paclitaxel, Vascular access

\section{Introduction}

Arteriovenous grafts (AVG) are frequently used for hemodialysis vascular access in patients who do not have vessels suitable for creation of a native arteriovenous fistula (AVF). ${ }^{1}$ In the United States, about $27-28 \%$ of patients undergoing hemodialysis have an AVF, whereas more than $40 \%$ have an AVG implanted $^{2}$. Hemodialysis vascular access dysfunction (HVAD) is a major problem of AVGs and can result from the aggressive development of venous neointimal hyperplasia. ${ }^{3}$ Much effort has been made to maintain the patency of grafts by preventing stenosis or thrombosis in venous anastomosis sites, but there is no effective therapeutic intervention for HVAD of AVG.

Another problem with AVG is significant tissue swelling and pain which may be due to changes in local hemodynamics and soft tissue injuries associated with inflammation. Therefore, ePTFE grafts cannot be routinely cannulated for at least two weeks after placement, until swelling has reduced enough to allow palpation over the course of the graft. ${ }^{4}$

Recently, we reported that ePTFE grafts coated with paclitaxel prevented neointimal hyperplasia and stenosis of AVG, especially at the site of graft-venous anastomosis in a pig model. $^{5-8}$ We also hypothesized that paclitaxel-coated ePTFE grafts may reduce significant tissue swelling and pain by preventing soft tissue inflammation. In this study, we investigated the effects of paclitaxel-coated ePTFE grafts on inflammation surrounding the perigraft and intragraft tissues in a rabbit model.

\section{Materials and Methods}

Preparation of paclitaxel-coated ePTFE grafts. In previous studies, coiled and carbon-lined grafts (IMPRA F4006C, BARD Peripheral Vascular, Inc., USA) were used to test the efficacy of paclitaxel coating. ${ }^{5,7,8}$ Noncoiled and noncarbon-lined grafts (IMPRA 40S06, BARD Peripheral Vascular, Inc., USA) were used to test the toxicity of paclitaxel as a function of the amount of coating. As described in these studies, paclitaxel-coated ePTFE vascular grafts were made in a single dipping method using paclitaxel (Genexol ${ }^{\circledR}$, Samyang Genex Inc., Korea) and ePTFE vascular grafts. The grafts were $6 \mathrm{~mm}$ in diameter and $400 \mathrm{~mm}$ in length.

Briefly, paclitaxel was dissolved in acetone (Fisher Scientific, Korea) to a concentration of either $1.0 \mathrm{mg} / \mathrm{mL}$ or $2.0 \mathrm{mg} / \mathrm{mL}$ in polypropylene tubes. ePTFE vascular grafts were dipped into one of these solutions and incubated for 30 minutes at $37{ }^{\circ} \mathrm{C}$ in a hybridization incubator (FinePCR, Combi-H, Korea). Paclitaxel-coated ePTFE grafts were then air-dried and maintained under vacuum for four hours to completely remove solvent. Paclitaxel-coated grafts were sterilized in ethylene oxide gas before use.

In vitro release kinetics. For the in vitro release test, phosphate-buffered saline (PBS, pH 7.4) containing $0.05 \%$ (w/v) Tween-20 (Hayashi Pure Chemical Ind., Ltd, Japan), a non-ionic surfactant, was used as the drug release medium.

Paclitaxel-coated vascular grafts of $3 \mathrm{~cm}$ length were soaked 
in polypropylene tubes with $5 \mathrm{~mL}$ of release medium and shaken for 30 minutes at $37^{\circ} \mathrm{C}$ in a hybridization incubator. At designated time points over the course of 21 days, release medium was removed completely from the tubes and analyzed.

The solution was analyzed by HPLC (Agilent 1200 Series, USA) using a $4.6 \times 150 \mathrm{~mm} \mathrm{C18}$ reverse-phase column, and a UV detector set at $227 \mathrm{~nm}$. The mobile phase used was water: acetonitrile $(50: 50 \mathrm{v} / \mathrm{v})$ under isocratic condition at a flow rate of $0.8 \mathrm{~mL} / \mathrm{min}$, where the paclitaxel elution peak occurred at $9.5 \mathrm{~min}$.

Experimental animals and operative technique. Drug-coated vascular grafts were placed on subcutaneous tissue of 12 male rabbits (New Zealand White) that were in good health weighing $2,500 \pm 200 \mathrm{~g}$. The animals were maintained in standard animal care facilities at Samsung Biomedical Research Institute. Each operating procedure was in conformity with the Guidelines for the Care and Use of Laboratory Animals (NIH publication No. 85-23, revised 1996). Paclitaxel-free control grafts, paclitaxelcoated grafts of low concentration $\left(0.61 \mathrm{ug} / \mathrm{mm}^{2}\right)$ and paclitaxelcoated grafts of high concentration $\left(1.15 \mathrm{ug} / \mathrm{mm}^{2}\right)$ were tested simultaneously in the 12 rabbits.

All rabbits were anesthetized with an intramuscular injection of ketamine $(35 \mathrm{mg} / \mathrm{kg}$ ) and xylazine $(5 \mathrm{mg} / \mathrm{kg})$. After shaving hair, surgical sites on the backs of the rabbits were sterilized with povidone. For each type of graft, two linear incisions about $2 \mathrm{~cm}$ in length were made with a sterile scalpel on the dorsal outer skin region, $5 \mathrm{~cm}$ apart from each other. Three types of $5 \mathrm{~cm}$ vascular grafts were placed laterally, separated by about $5 \mathrm{~cm}$, passing through the incisions. The grafts were fixed to the surrounding tissue with a 6 - 0 polypropylene suture. After implantation, incisions were closed and cefazolin $(11 \mathrm{mg} / \mathrm{kg})$ was injected intramuscularly to prevent infection. Six rabbits were maintained for one week before explantation, while the remaining six rabbits were anesthetized at two weeks by injecting $\mathrm{KCl}$ through the ear vein. Grafts and adjacent tissues were excised and immersed in 10\% natural buffered formalin (NBF) for at least 24 hours.

Tissue preparation and morphometric analysis. The excised specimens were fixed in $10 \%$ buffered formalin and embedded in paraffin. Five micrometer thick sections of grafts and adjacent tissues were prepared perpendicular to the graft. All sections were stained with hematoxylin and eosin (H\&E).

Immunohistochemical staining for alpha-smooth muscle actin ( $\alpha$-SMA) was performed to characterize the stromal cells on the sections from grafts. Five-micron-thick sections were deparaffinized and incubated with primary antibody against $\alpha$ SMA (1 : 400 dilution, Abcam PLC, UK) and then with Vectastain ABC kits (Vector, USA) according to the manufacturer's instructions.

A microscope (BX50F, Olympus Digital Camera System, Japan) was used to count perigraft and intragraft inflammatory cells. The number of infiltrating inflammatory cells per unit area was counted in five high power fields $(\times 400)$ using Image J software. Three independent observers counted the inflammatory cells on each slide and graded stromal cell proliferation semiquantitatively as negative ( 0 ), equivocal (1), moderate (2) and marked (3). Mean of each count or scores were calculated.

Statistics. All data are presented as mean \pm SD. The differ- ences in parameters between the control and paclitaxel-coated group were examined using the Page test and Mann-Whitney $U$ test (SPSS 12.0 for Windows, SPSS Inc.). P-values of less than 0.05 were considered statistically significant.

\section{Results and Discussion}

In vitro release test. Under $1 \mathrm{mg} / \mathrm{mL}$ and $2 \mathrm{mg} / \mathrm{mL}$ paclitaxel coating conditions, the loading amounts of paclitaxel on carbonlined grafts were $0.67 \mathrm{ug} / \mathrm{mm}^{2}$ and $1.38 \mathrm{ug} / \mathrm{mm}^{2}$, and on noncarbon-lined grafts was $0.61 \mathrm{ug} / \mathrm{mm}^{2}$ and $1.15 \mathrm{ug} / \mathrm{mm}^{2}$, respectively. In vitro paclitaxel release kinetics revealed that, at similar coating levels, the drug-eluting rate of carbon-lined grafts was lower than that of noncarbon-lined grafts (Figure 1). The retention of paclitaxel on carbon-lined grafts may be increased because paclitaxel is more strongly attached to the hydrophobic surface of carbon-lined grafts through increased electrostatic forces.

Animal experiments and pathologic analysis. Necrosis and edema were not found by gross examination in any excised perigraft tissues. Blood vessel formation in the surrounding tissue of implanted paclitaxel-free control grafts was detected two
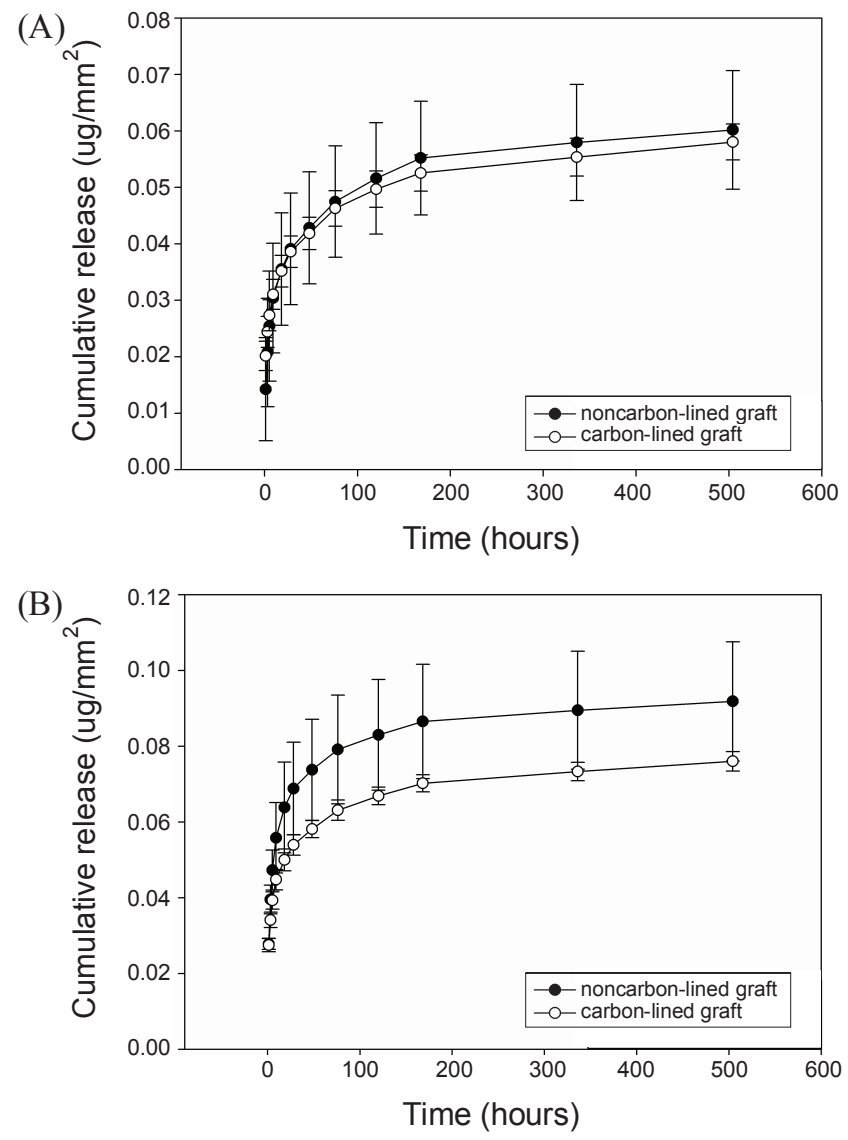

Figure 1. (A) In vitro cumulative release profiles of paclitaxel from noncarbon-lined grafts (closed circle) and carbon-lined grafts (open circle) in the $1 \mathrm{mg} / \mathrm{mL}$ coating condition. Data reflect the mean of five experiments. (B) In vitro cumulative release profiles of paclitaxel from noncarbon-lined grafts (closed circle) and carbon-lined grafts (open circle) in the $2 \mathrm{mg} / \mathrm{mL}$ coating condition. Data reflect the mean of five experiments. 

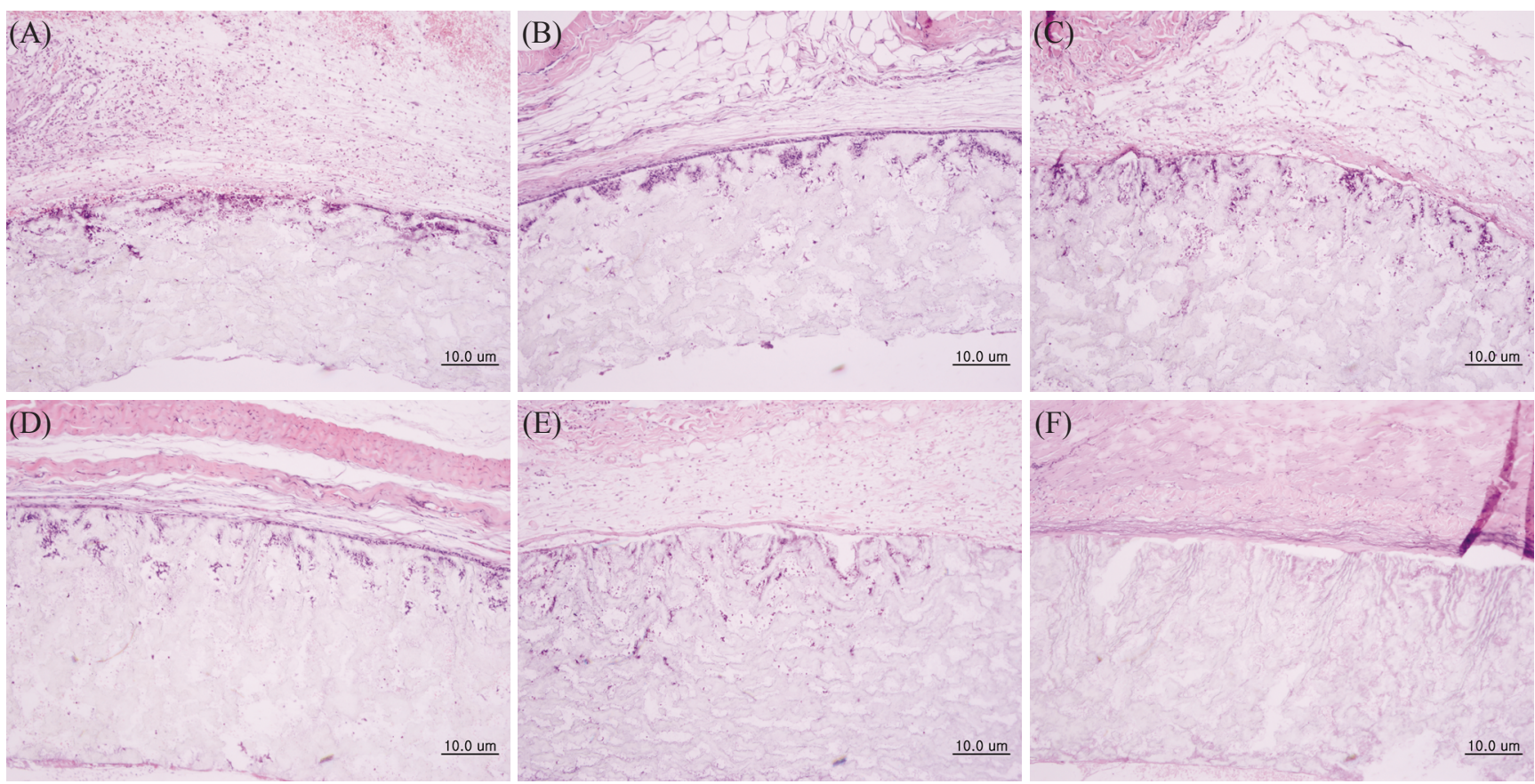

Figure 2. Light micrographs of implanted grafts and surrounding tissue under varied paclitaxel concentrations. The number of inflammatory cells in the control grafts (A, B) was greater than those in paclitaxel- coated grafts of low concentration (C, D) and high concentration (E, F). The number of inflammatory cells was greater in samples obtained one week after implantation (A, C, E) compared with two weeks after implantation (B, D, F). Higher paclitaxel concentrations correlated to narrower stromal cell layers (adjacent line of intragraft and perigraft). Stromal cell layer was thicker at two weeks than at one week (H\&E staining, original magnification X100).

weeks after operation, but blood vessels were not seen clearly in either paclitaxel-coated group (data not shown).

The tissue reaction observed after implantation of ePTFE vascular grafts may be similar to typical wound healing processes, which consist of inflammation, cellular proliferation, connective tissue formation, wound contraction, and wound remodeling. ${ }^{9}$ The intensity and duration of the inflammatory response is a major determinant of the biocompatibility of biomaterial implantation. ${ }^{10}$

Perigraft inflammation, graft wall inflammation and stromal cell proliferation were evaluated by microscopy (Figure 2). As shown in Figure 3, the number of inflammatory cells decreased significantly with increasing concentration of paclitaxel coating at both intragraft $(\mathrm{P}=0.0017$ at one week and $\mathrm{P}=0.0017$ at two weeks $)$ and perigraft $(\mathrm{P}=0.0001$ at one week and $\mathrm{P}=0.0054$ at two weeks), according to the results of the 'Page test'. The number of inflammatory cells was decreased in tissue excised at two weeks compared with tissue excised at one week after implantation at the same paclitaxel concentration $(\mathrm{P}=0.0001$ at intragraft and $\mathrm{P}=0.0018$ at perigraft, as indicated by mix model analysis).

During the initial phase of healing, inflammatory cells such as neutrophils and eosinophils arrive at the wound site followed by lymphocytes and circulating macrophages. In control grafts, there were numerous inflammatory cells in the tissue surrounding the graft and graft wall. However, inflammatory cells decreased markedly with increasing concentrations of paclitaxel. Over the time course, the number of inflammatory cells was highest when observed one week after implantation and then significantly diminished by two weeks for grafts under the same conditions. Intragraft inflammatory cells were reduced by more than $50 \%$ at two weeks after implantation for each type of graft, while perigraft inflammatory cells had diminished by less than $50 \%$ at two weeks. These observations suggest that paclitaxel suppressed internal and external inflammation of the graft, in proportion to the coating concentration of the drug.

Inflammation around graft causes significant tissue swelling and pain, which can delay cannulation and sometimes be severe enough to cause morbidities. Goldstein and colleagues reported a case of median nerve compression from a previously undiscovered lipoma exacerbated by perigraft swelling after the implantation of a PTFE graft. ${ }^{11}$ Because perigraft edema occurs in almost all cases of ePTFE graft implantation, the first puncture of a graft is routinely started more than 14 days after implantation. $^{12}$ Therefore, the implantation of paclitaxel-coated vascular grafts may significantly reduce morbidities and provide a novel advantage for early first punctures of grafts through prevention of perigraft edema.

Microvessel formation was observed within the subcutaneous tissue of control grafts, with more severe inflammatory reactions than paclitaxel-coated grafts. Microvessel formation from existing blood vessels may be processed by inflammatory cells that are known to affect angiogenesis. ${ }^{13-15}$ Otherwise, the inflammatory response could also be inhibited by reduced microvessel formation with paclitaxel-coated grafts.

Using H\&E staining, we observed not only an inflammatory reaction but also stromal cell proliferation. Cell layers composed of spindle to ovoid cells (stromal cells) were noted in the interface between implanted grafts and subcutaneous connective tissue. These stromal cells were observed ingrowing into the 
(A)

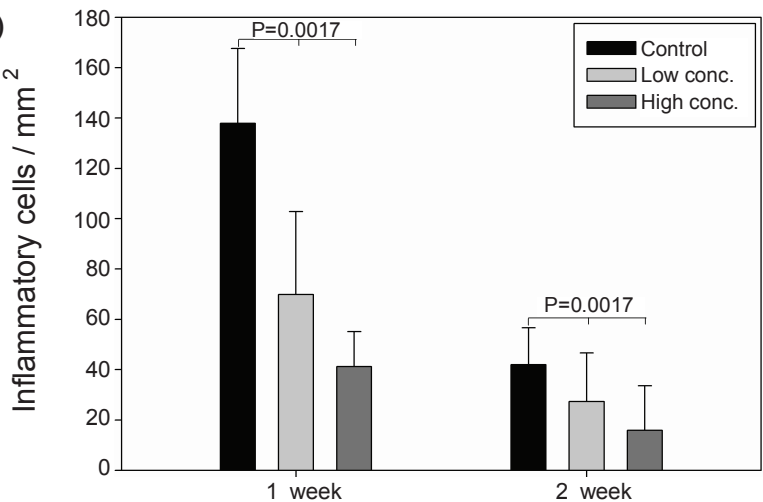

(B)

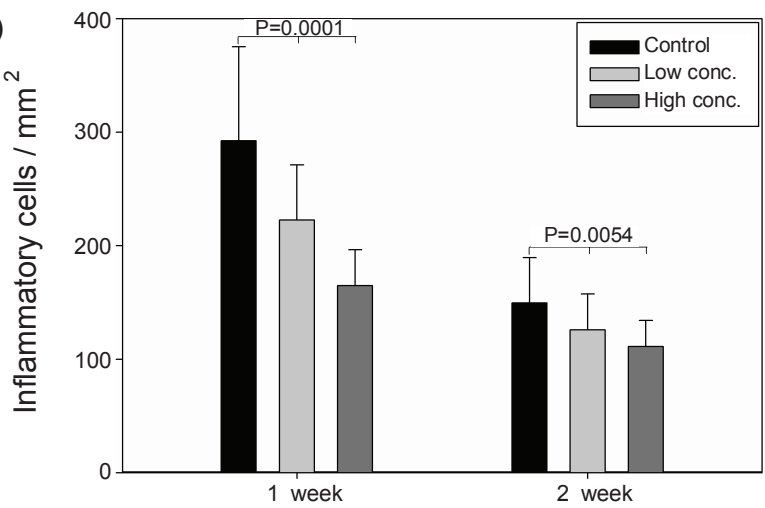

Figure 3. The number of inflammatory cells per unit area in relation to the paclitaxel coating amount on each graft. (A) Intragraft inflammatory cells were significantly reduced with increasing concentrations of paclitaxel coating $(\mathrm{P}=0.0017$ at one week, $\mathrm{P}=0.0017$ at two weeks, analyzed by Page test). There were fewer intragraft inflammatory cells in tissue excised at two weeks than one week after implantation at the same paclitaxel concentration ( $\mathrm{P}=0.0001$, Analysis Using Mixed Model). (B) Perigraft inflammatory cells were diminished with increasing concentrations of paclitaxel coating $(\mathrm{P}=0.0001$ at one week, $\mathrm{P}=$ 0.0054 at two weeks, analyzed by Page test). There were fewer perigraft inflammatory cells in tissue excised at two weeks than one week after implantation at the same paclitaxel concentration $(\mathrm{P}=0.0018$, Analysis Using Mixed Model).

interstices of grafts (Fig. 4-B). We tested whether myofibroblast occurred in the stromal cell layer by immunohistochemistry with $\alpha$-smooth muscle actin ( $\alpha$-SMA) antibody. ${ }^{16} \alpha$-SMA is expressed in contractile vascular smooth muscle cells (VSMCs), but also expressed during the period when fibroblast phenotypes change to myofibroblast. ${ }^{17,18}$ Therefore, cells that are positive for $\alpha$-SMA and have a stellate form are considered to be myofibroblasts. The ability of myofibroblasts to produce a contractile force is essential for tissue contracture. ${ }^{19}$ During the wound contraction phase, myofibroblasts are known to enter the graft interstices and begin to pull adjacent structures closer together. ${ }^{20}$ Stromal cell proliferation was marked in the control group (Fig. 2 A-B) while stromal cell proliferation was significantly reduced with increasing paclitaxel concentration in the paclitaxel-coated groups (Fig. 2 C-F). Within each group, proliferation of stromal cells was greater at two weeks than one week. The degree of stromal cell proliferation in the control graft was significantly greater than that of low dose paclitaxel-coated grafts and high
(A)

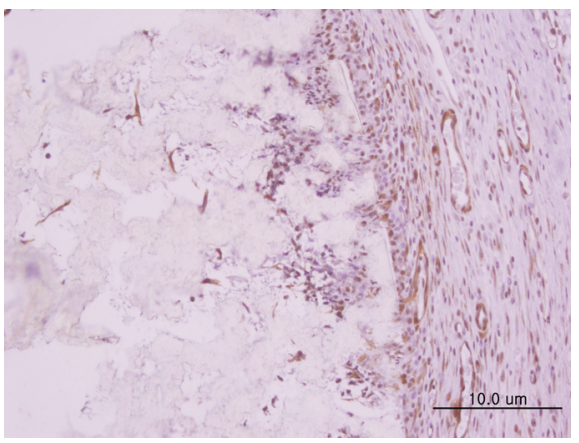

(B)

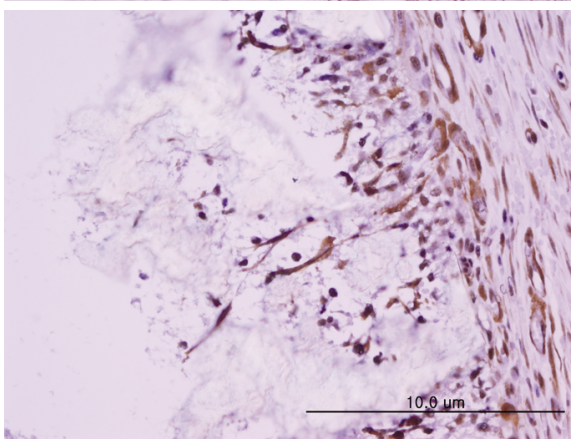

Figure 4. Stromal cell infiltration into graft interstices. $\alpha$-smooth muscle actin antibody staining confirmed focal presence of myofibroblasts $(+)$. (A) Immunohistochemistry, original magnification X200. (B) Immunohistochemistry, original magnification X400.

dose paclitaxel-coated grafts, but there was no statistically significant difference between the low and high concentrations of paclitaxel-coated grafts in each week. There was focal positivity for $\alpha$-SMA in surrounding perigraft and intragraft tissues in the control group (Fig. 4-A), which gradually infiltrated from the perigraft into the intragraft (Fig. 4-B). Interestingly, paclitaxel coating significantly reduced stromal cell proliferation.

Other studies have also reported that fibroblasts originating from the perigraft region infiltrate into the intragraft. ${ }^{21-22}$ Our findings indicate that stromal cells that infiltrate into the graft interstices may differentiate into myofibroblasts, so that, upon firm attachment to the graft wall, they may result in stable graft placement by participating in a contractile reaction of grafts (Fig. 4-B). The observation of fibrous encapsulation of vascular grafts, occurring through mechanisms such as myofibroblast infiltration, may suggest that biomedical materials are healed within tissue around an implanted vascular graft. Around paclitaxel-coated graft, in contrast, the stromal cell layer was thinner and myofibroblasts were rarely detected and separated from surrounding tissue. The suppression of stromal cell proliferation by paclitaxel may retard the graft healing process, including fibrous encapsulation and furthermore, attenuate tissue-to-graft incorporation. ${ }^{23-24}$ Therefore, coated amount of paclitaxel and drug release rate are very important for graft healing. The paclitaxelcoated graft with low dose showed less inhibitory effect on stromal cell proliferation than graft with high dose. And, as the paclitaxel concentrations in arteriovenous grafts may rapidly decrease due to washout by blood flow, the influence of paclitaxel on the fibrosis would be attenuated with time after graft construction as the real clinical situation. 


\section{Conclusions}

In summary, paclitaxel coated-ePTFE grafts were found to effectively suppress inflammation around the implanted grafts. This may reduce morbidities and allow earlier cannulation by preventing soft tissue swelling and pain.

Acknowledgments. This study was supported by a grant of the Korea Healthcare technology R\&D Project, Ministry of Health \& Welfare, Republic of Korea (A080349). This work was supported by the Samsung Biomedical Research Institute grant.

\section{References}

1. Konner, K. Blood Purif. 2006, 24(3), 287.

2. Am. J. Kidney Dis. 2003, 41(4 Suppl 2), v.

3. Hakim, R.; Himmelfarb, J. Kidney Int. 1998, 54(4), 1029.

4. Am. J. Kidney Dis. 2001, 37(1 Suppl 1), S137.

5. Lee, B. H.; Lee, J. E.; Lee, K. W.; Nam, H. Y.; Jeon, H. J.; Sung, Y. J.; Kim, J. S.; Lim, H. J.; Park, J. S.; Ko, J. Y.; Kim, D. J. Nephrol. Dial. Transplant. 2007, 22(10), 2800.

6. Lim, H. J.; Nam, H. Y.; Lee, B. H.; Kim, D. J.; Ko, J. Y.; Park, J. S. Biotechnol. Prog. 2007, 23(3), 693.

7. Lee, B. H.; Nam, H. Y.; Kwon, T.; Kim, S. J.; Kwon, G. Y.; Jeon, H. J.; Lim, H. J.; Lee, W. K.; Park, J. S.; Ko, J. Y.; Kim, D. J. Nephrol. Dial. Transplant. 2006, 21(9), 2432.

8. Nam, H. Y.; Kim, D. J.; Lim, H. J.; Lee, B. H.; Baek, I.; Park, S. H.; Park, J. S. Bull. Korean Chem. Soc. 2008, 29(2), 422.
9. Orgill, D.; Demling, R. H. Crit. Care Med. 1988, 16(9), 899.

10. Busuttil, S. J.; Drumm, C.; Plow, E. F. BC Decker Inc.: 2005; p 230.

11. Goldstein, L. J.; Helfend, L. K.; Kordestani, R. K. Neurosurgery 2002, 50(2), 412.

12. Matsuda, H.; Miyazaki, M.; Oka, Y.; Nakao, A.; Choda, Y.; Kokumai, Y.; Kunitomo, K.; Tanaka, N. Artificial Organs 2003, 27(8), 722 .

13. Sunderkotter, C.; Steinbrink, K.; Goebeler, M.; Bhardwaj, R.; Sorg, C. J. Leukoc. Biol. 1994, 55(3), 410.

14. Sunderkotter, C.; Goebeler, M.; Schulze-Osthoff, K.; Bhardwaj, R.; Sorg, C. Pharmacol. Ther. 1991, 51(2), 195.

15. Salzmann, D. L.; Kleinert, L. B.; Berman, S. S.; Williams, S. K. Cardiovasc. Pathol. 1999, 8(2), 63.

16. Chaponnier, C.; Gabbiani, G. J. Pathol. 2004, 204(4), 386.

17. Tomasek, J. J.; McRae, J.; Owens, G. K.; Haaksma, C. J. Am. J. Pathol. 2005, 166(5), 1343.

18. Jin, D.; Ueda, H.; Takai, S.; Muramatsu, M.; Furubayashi, K.; Ibaraki, T.; Kishi, K.; Katsuoka, Y.; Miyazaki, M. Life Sciences 2007, 81(16), 1291.

19. Tomasek, J. J.; Gabbiani, G.; Hinz, B.; Chaponnier, C.; Brown, R. A. Nat. Rev. Mol. Cell Biol. 2002, 3(5), 349.

20. Storch, M.; Perry, L. C.; Davidson, J. M.; Ward, J. J. Surg. Infect (Larchmt) 2002, 3 Suppl 1, S89.

21. Stronck, J. W.; van der Lei, B.; Wildevuur, C. R. J. Thorac. Cardiovasc. Surg. 1992, 103(1), 146.

22. Brewster, L. P.; Bufallino, D.; Ucuzian, A.; Greisler, H. P. Biomaterials 2007, 28(34), 5028.

23. Axel, D. I.; Kunert, W.; Goggelmann, C.; Oberhoff, M.; Herdeg, C.; Kuttner, A.; Wild, D. H.; Brehm, B. R.; Riessen, R.; Koveker, G.; Karsch, K. R. Circulation 1997, 96(2), 636.

24. Eisinger, D. R.; Sheil, A. G. Surg. Gynecol. Obstet. 1985, 160(2), 135 